International Journal of Bifurcation and Chaos, Vol. 12, No. 6 (2002) 1395-1402

(c) World Scientific Publishing Company

\title{
CONTROLLED PROJECTIVE SYNCHRONIZATION IN NONPARTIALLY-LINEAR CHAOTIC SYSTEMS
}

\author{
DAOLIN XU and ZHIGANG LI \\ Center for Advanced Numerical Engineering Simulation, \\ School of Mechanical and Production Engineering, \\ Nanyang Technological University, Singapore 639798
}

Received May 7, 2001; Revised June 25, 2001

\begin{abstract}
Projective synchronization (PS), in which the state vectors synchronize up to a scaling factor, is usually observable only in partially linear systems. We show that PS could, by means of control, be extended to general classes of chaotic systems with nonpartial linearity. Performance of PS may also be manipulated by controlling the scaling factor to any desired value. In numerical experiments, we illustrate the applications to a Rössler system and a Chua's circuit. The feasibility of the control for high dimensional systems is demonstrated in a hyperchaotic system.
\end{abstract}

Keywords: Control; project synchronization; chaotic system.

\section{Introduction}

In light of the potential application in secure communications [Cuomo \& Oppenheim, 1993; Hayes et al., 1994; Kovarev et al., 1992; Parlitz, 1996], synchronization in coupled chaotic systems has attracted much interest since Pecora and Carroll first introduced this important dynamical phenomenon [Pecora \& Carroll, 1990]. However, "synchronization" is by far sufficient to describe the mutually cooperative behavior between the coupled systems.

Different types of synchronization phenomena have been observed in a variety of chaotic systems. The occurrence of a particular type of synchronization may depend on a coupling scheme and the structure of the underlying dynamical system considered. For two coupled chaotic oscillators [Rosenblum et al., 1996], phase synchronization could occur in the manner that the phases $\theta_{1,2}$ are locked with $n \theta_{1}-m \theta_{2}=$ constant while the amplitudes can be very different. In coupled chaotic map lattices, the global activity of the lattices can be characterized by phase synchronization but the local activity of each map remains unsynchronized. Such collective behavior is referred to as weak synchronization [Roman et al., 1998]. Identical synchronization [Pecora \& Carroll, 1990; Hayes et al., 1993 is the most typical form of chaotic synchronization often observable in two identical systems. It is characterized by the feature that the phases are synchronized and the amplitudes tend to be the same. When the parameters of the two-coupled systems do not match, or even the two coupled systems belong to different classes [Kocarev \& Parlitz, 1996], there is a general relation between the states of the two subsystems. This synchronous relation could be expressed by a smooth, invertible function, known as generalized synchronization [Rulkov et al., 1995]. In coupled partially linear systems, Mainieri and Rehacek [1999] reported that the two identical systems (drive and response) could be synchronized up to a scaling factor. This type of chaotic synchronization is referred to as projective synchronization (PS). Although there exists a linear relation between the coupled systems, PS could not be classified as generalized synchronization because the 
response system of PS is not asymptotically stable [Kocarev \& Parlitz, 1996; Li \& Xu, 2001].

Projective synchronization is interesting because of the proportionality of the dynamical scales and the invariance of chaotic characteristics (such as Lyapunov exponents and fractal dimensions) between the states of the two-coupled systems. The feature of proportionality enables us to duplicate a topologically identical system in distinct scales through a PS process. PS could be considered as a candidate for secure communications with an additional advantage of using an adjustable scaling factor, which will be reported elsewhere.

In the scenario of PS, the evolutionary pattern governed by the response system is similar to that governed by the drive system. However the amplitude of the response system is a scalar multiple of that of the drive system in a phase space. This scalar multiple is referred to as scaling factor. In an article by Mainieri and Rehacek [1999], the authors explained the mechanism of the occurrence of PS in three-dimensional systems. In fact, PS is determined by the inherent partial linearity of the systems. Investigation pertaining to the stability criterion of PS has also been recently conducted in [Li \& Xu, 2001] stating that PS occurs if the trace of the Jacobian matrix of the subsystem is negative. This statement implies that PS could occur even if some Lyapunov exponents are positive. In other words, the widely accepted condition that all conditional Lyapunov exponents should be negative [Pecora \& Carroll, 1990] for chaotic synchronization is not necessary. Note that the resulting dynamics in PS is hardly predictable [Xu, 2001] because the scaling factor is dependent on the initial conditions and the underlying chaotic dynamics. To achieve a desirable performance of PS, some control methods have been developed [Xu, 2001; Xu \& Li, 2001] to manipulate the scaling factor. It is thus possible to adjust (amplify or reduce) the scale of the coupled dynamics with small controls. Control of PS may provide more flexibility to promote PS in various applications.

The availability of PS seems limited to the class of partially linear chaotic systems. Although the projective nature was also observed in the Lorenz system with a specific coupling scheme [Kocarev \& Parlitz, 1996], the Lorenz system actually possesses partial linearity. It is natural to think if it is possible to extend PS to more general classes of nonlinear systems rather than the class of partial linearity. In this paper, we introduce a control approach to produce PS in nonlinear chaotic systems. In addition, the control method can be used to direct the scaling factor to any preferred value for any desirable performance of synchronized dynamics. The control approach is derived from the Lyapunov stability theory and thus the control is globally stable.

\section{Projective Synchronization}

We firstly elaborate PS in partially linear systems such as the Lorenz system. A partially linear system is defined as the system in which the state vector consists of two parts $(\mathbf{u}, z)$. Vector $\mathbf{u}$ is linearly related to its time derivative $\dot{\mathbf{u}}$, while variable $z$ is nonlinearly related to the variables in $\mathbf{u}$, as given by

$$
\begin{aligned}
\dot{\mathbf{u}} & =\mathbf{A}(z) \cdot \mathbf{u}, \\
\dot{z} & =f(\mathbf{u}, z) .
\end{aligned}
$$

Projective synchronization may be observable when two identical systems (1) are coupled through the variable $z$ in the form

$$
\begin{aligned}
\dot{\mathbf{u}}_{m} & =\mathbf{A}(z) \cdot \mathbf{u}_{m}, \\
\dot{z} & =f\left(\mathbf{u}_{m}, z\right), \\
\dot{\mathbf{u}}_{s} & =\mathbf{A}(z) \cdot \mathbf{u}_{s} .
\end{aligned}
$$

The subscripts $m$ and $s$ stand for the master (drive) and slave (response) systems respectively. In the coupled system (2), the drive system evolves independently, while the response system is governed by the drive system through the variable $z$. With certain parametrical settings, PS takes place and exhibits the dynamical feature that the two state vectors tend to a proportional relation, i.e. $\lim _{t \rightarrow \infty}\left\|\alpha \mathbf{u}_{m}-\mathbf{u}_{s}\right\|=0$, where $\alpha$ is a constant scaling factor. The sign of $\alpha$ is determined by the phase difference, $\omega \in(-\pi, \pi]$, between the drive and response systems. When $t \rightarrow \infty, \omega= \pm \pi$, then $\alpha$ is negative. When $t \rightarrow \infty, \omega=0, \alpha$ is positive.

We provide an example in Fig. 1 to illustrate the dynamical evolution of projective synchronization in the Lorenz system [Lorenz, 1963]

$$
\begin{aligned}
& \dot{x}=\sigma(y-x), \\
& \dot{y}=(\mu-z) x-y, \\
& \dot{z}=x y-\rho z .
\end{aligned}
$$

Figure 1 shows projective synchronization in which the drive system and response system move in the same phase $\left(\theta_{s}-\theta_{m}=0, \alpha<0\right)$. Figure $1(\mathrm{a})$ 


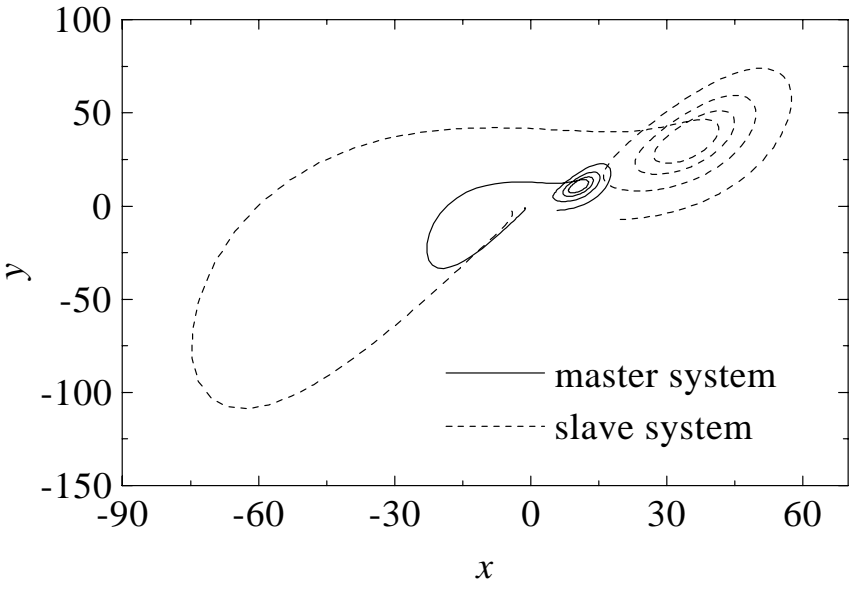

(a)

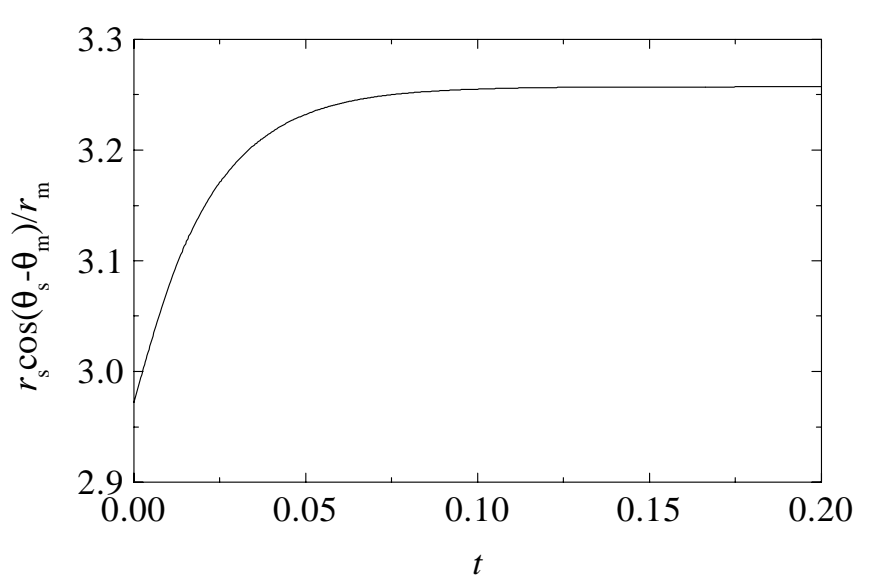

(b)

Fig. 1. Projective synchronization for the Lorenz system $(\sigma=10, \mu=60$ and $\rho=8 / 3)$ with the initial condition $\left\{x_{m}\right.$, $\left.y_{m}, z, x_{s}, y_{s}\right\}=\{0.1,0.2,0.1,0.4,0.5\}$; (a) two synchronized trajectories in $x-y$ plane; (b) the evolution of the ratio of $r_{s} \cos \left(\theta_{s}-\theta_{m}\right) / r_{m}$ which tends to a scaling factor $\alpha=3.257$ after a short transient (where $r=\sqrt{x^{2}+y^{2}}, \theta_{s}=\operatorname{tg}\left(y_{s} / x_{s}\right)$ and $\left.\theta_{m}=\operatorname{tg}\left(y_{m} / x_{m}\right)\right)$.

depicts the trajectory evolution (where the transient evolution is discarded) in $x-y$ plane and Fig. 1(b) the evolution of the ratio $r_{s} \cos \left(\theta_{s}-\right.$ $\left.\theta_{m}\right) / r_{m}$, where $r=\sqrt{x^{2}+y^{2}}$ is the norm of the state vector and $\theta=\operatorname{tg}(y / x)$ the phase angle.

When PS happens, the angular difference $\omega=$ $\theta_{s}-\theta_{m}$ converges to either 0 or $\pi$ and $r_{s} \cos \left(\theta_{s}-\right.$ $\left.\theta_{m}\right) / r_{m}$, tends to the scaling factor $\alpha$. We remark that the form $\mathbf{u}_{s}=\alpha \mathbf{u}_{m}$ is a solution of PS for partially linear systems, but usually not a solution for nonpartially linear systems. To realize PS in a coupled nonpartially linear chaotic system, a control may be necessary to force the coupled system to perform the projective nature.

\section{Control Method and Numerical Experiments}

Now we intend to extend the PS feature to general nonlinear systems rather than partially linear systems by employing a control method. Our aim is that when the drive system generates a chaotic dynamics, the response system can, by control, track and synchronize the driving dynamics with a specified scaling factor. To perform PS in a coupled nonlinear system, we devise our control scheme based on the following considerations. (a) The feedback control will be set to the response system only. This is because the originality of the chaotic signals from the drive should not be altered by any artificial interruption (i.e. control signals). Considering a potential application of secure communications in which information is masked by a driving dynamics, we only need to control the response system to synchronize the response dynamics with the driving dynamics. (b) The controlled synchronization should possess a global stability so that the synchronization process will be free of the sensitivity to initial conditions. In other words, once control is activated, the response system can be immediately controlled to synchronize with the drive system whatever be the initial states.

Based on the above considerations, we introduce a control vector $\mathbf{v}$ to the response system, in the form

$$
\begin{aligned}
\dot{\mathbf{u}}_{m} & =\mathbf{g}\left(\mathbf{u}_{m}, z\right), \\
z & =f(\mathbf{u}, z), \\
\dot{\mathbf{u}}_{s} & =\mathbf{g}\left(\mathbf{u}_{s}, z\right)+\mathbf{v},
\end{aligned}
$$

where $\mathbf{g}$ is a nonlinear function vector with respect to $\mathbf{u}$ and $z$. The control vector $\mathbf{v}$ has the same dimension as $\mathbf{u}$. Note that without control (i.e. $\mathbf{v}=0$ ), the dynamical states $\mathbf{u}_{m}$ and $\mathbf{u}_{s}$ defined by the coupled system (4) evolve with no patterns, and PS does not occur. Our purpose is to find an appropriate control vector $\mathbf{v}$ for the system (4) so that PS happens with a desired scaling factor $\alpha^{*}$, i.e. $\lim _{t \rightarrow \infty}\left\|\alpha^{*} \mathbf{u}_{m}-\mathbf{u}_{s}\right\|=0$.

Concerning the development of a control algorithm with the property of global convergence, we employ the Lyapunov stability theory by defining a proper Lyapunov function that must be globally stable. To constitute the function, we introduce an error vector $\mathbf{e}(t)=\alpha^{*} \mathbf{u}_{m}(t)-\mathbf{u}_{s}(t)$ according to the form of PS solution, where the components $e_{i}(t)=\alpha^{*} u_{m, i}(t)-u_{s, i}(t)$, and the variables $u_{m, i}(t)$ 
and $u_{s, i}(t)$ are the $i$ th components of $\mathbf{u}_{m}$ and $\mathbf{u}_{s}$ respectively. If the control vector $\mathbf{v}$ is well defined such that when $\lim _{t \rightarrow \infty} \mathbf{e}(t)=0$, PS will happen in the coupled system (4). We consider a Lyapunov function in the form of

$$
V=\frac{1}{2} \mathbf{e}^{T} \mathbf{e}=\frac{1}{2} \sum_{i=1}^{n} e_{i}^{2},
$$

where the superscription $T$ is a transpose. If the Lyapunov function (5) satisfies the conditions

$$
\begin{cases}V(\mathbf{e})>0 & \text { if } \mathbf{e} \neq 0 \\ V(\mathbf{e})=0 & \text { if } \mathbf{e}=0\end{cases}
$$

and

$$
\left\{\begin{array}{ll}
\dot{V}(\mathbf{e})<0 & \text { if } \mathbf{e} \neq 0 \\
\dot{V}(\mathbf{e})=0 & \text { if } \mathbf{e}=0
\end{array},\right.
$$

the error vector $\mathbf{e}(t)$ will asymptotically tend to zero such that $\lim _{t \rightarrow \infty}\left\|\alpha^{*} \mathbf{u}_{m}-\mathbf{u}_{s}\right\|=0$. It implies the occurrence of PS at the scaling factor of $\alpha^{*}$. The first condition (6) can be satisfied obviously. For the second condition (7), we need to evaluate $\dot{V}$ by referring to system (4), and thus we have

$$
\begin{aligned}
\dot{V} & =\sum_{i=1}^{n} e_{i} \dot{e}_{i}=\mathbf{e}^{T} \dot{\mathbf{e}} \\
& =\mathbf{e}^{T}\left(\alpha^{*} \mathbf{g}\left(\mathbf{u}_{m}, z\right)-\mathbf{g}\left(\mathbf{u}_{s}, z\right)-\mathbf{v}\right) \\
& =\varphi(\mathbf{e}, \mathbf{v}) .
\end{aligned}
$$

In Eq. (8), the derivative $\dot{V}$ is, in fact, a function of the control vector $\mathbf{v}$. The sign of $\dot{V}$ can be determined by an appropriate selection of the control vector $\mathbf{v}$. If the control vector $\mathbf{v}$ is chosen in a way that results in $\dot{V}<0$ for $\mathbf{e} \neq 0$, then the coupled system (4) will perform PS with a scaling factor of $\alpha^{*}$ for any initial state. In fact, there is great flexibility in defining the control vector $\mathbf{v}$ to achieve a controlled PS. An explicit form of control depends on specific forms of the coupled system (4). In what follows, we will derive the control vector through the examples where the Rössler system, the Chua's circuit and a hyperchaotic system of high dimensions are considered to illustrate the implementations of the control.

To embody the control method, we apply the above idea to the Rössler system [Rössler, 1976] that is not a partially linear system if $z$ is used as the coupling variable, as in

$$
\begin{aligned}
& \dot{x}=b+x(y-c), \\
& \dot{y}=-x-z, \\
& \dot{z}=y+a z,
\end{aligned}
$$

which behaves chaotically with the settings $a=$ $0.398, b=2$ and $c=4$. In this system, $\mathbf{u}=(x, y)$, $\mathbf{e}=\left(e_{1}, e_{2}\right), \mathbf{v}=\left(v_{1}, v_{2}\right), z$ is the coupling variable and Eq. (8) reads

$$
\begin{aligned}
\dot{V}= & \left(\left(\alpha^{*}-1\right) b+\alpha^{*} x_{m} y_{m}-x_{s} y_{s}\right) e_{1}-c e_{1}^{2} \\
& +\left(1-\alpha^{*}\right) z e_{2}-e_{1} e_{2}-v_{1} e_{1}-v_{2} e_{2} .
\end{aligned}
$$

An option for $\dot{V}$ to be negative is that the control vector $\mathbf{v}$ may be defined as

$$
\left\{\begin{aligned}
v_{1}= & \left(\alpha^{*}-1\right) b+\alpha^{*} x_{m} y_{m} \\
& -x_{s} y_{s}+k_{1}\left(\alpha^{*} x_{m}-x_{s}\right) \\
v_{2}= & \left(1-\alpha^{*}\right) z-\left(\alpha^{*} x_{m}-x_{s}\right) \\
& +k_{2}\left(\alpha^{*} y_{m}-y_{s}\right)
\end{aligned}\right.
$$

According to the Lyapunov stability theory, the coupled Rössler system will produce PS under the control (11). The parameters $k_{1}$ and $k_{2}$ in Eq. (11) can be arbitrarily selected provided they satisfy the conditions (11). Substitution of Eq. (11) into Eq. (10), we have $V=-\left(k_{1}+c\right) e_{1}^{2}-k_{2} e_{2}^{2}$. Hence the dynamics of the two components of $\mathbf{e}$ are governed by $e_{1}=e_{10} \exp \left(-\left(k_{1}+c\right) t\right)$ and $e_{2}=e_{20} \exp \left(-k_{2} t\right)$ respectively, where the subscript " 0 " denotes initial conditions. It indicates that the preselected parameters $k_{1}$ and $k_{2}$ have an impact on the convergence of the control. In fact $k_{1}$ and $k_{2}$ define the convergence rates of the errors $e_{1}$ and $e_{2}$, respectively. The larger the values of $k_{1}$ and $k_{2}$, the faster is the convergence rate of the control. From Eq. (11), we can find that the control vector $\mathbf{v}$ does not vanish after PS happens. It is because the projective solution is not a solution of the original (uncontrolled) system.

Figure 2 shows two cases for the Rössler system, where PS is controlled to the desired scaling factors as $\alpha^{*}=5$ in Figs. 2(a) and 2(b) and $\alpha^{*}=-5$ in Figs. 2(c) and 2(d), respectively. The scaling factor can be chosen arbitrarily. Figures 2(a) and 2(c) depict the controlled trajectories of PS in the $x-y$ plane (transient evolution is not plotted here). For the case $\alpha^{*}=5$, the state vectors of the master system and the slave system evolve in the same phase angle, i.e. $\theta_{s}-\theta_{m}=0$; for the case $\alpha^{*}=-5$, the state vectors of the master system and the slave system evolve in the opposite direction, i.e. $\theta_{s}-\theta_{m}=\pi$. Figures 2(b) and 2(d) plot the evolutions of the ratio $r_{s} \cos \left(\theta_{s}-\theta_{m}\right) / r_{m}$, which asymptotically converges to a desired scaling factor. The effects on the control resulting from the variations of $k_{1}$ and $k_{2}$ are 


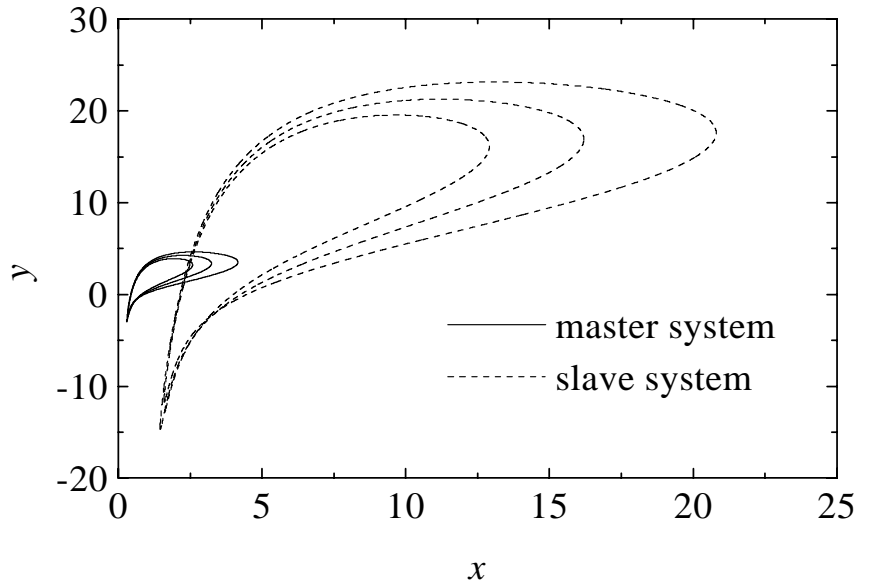

(a)

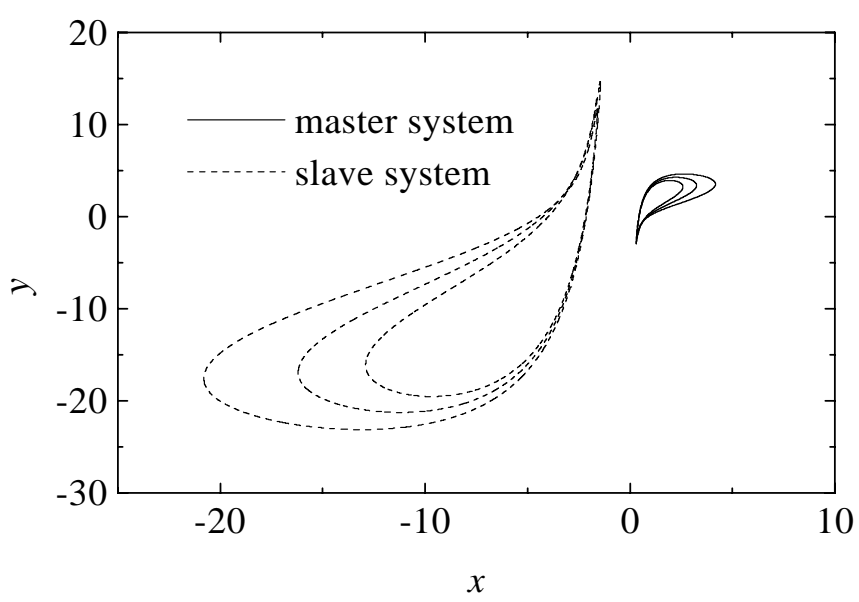

(c)

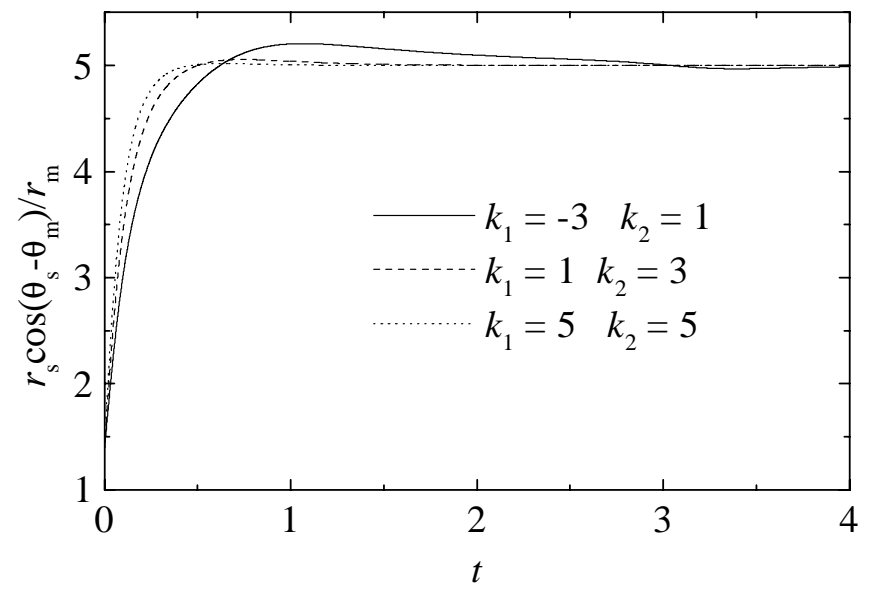

(b)

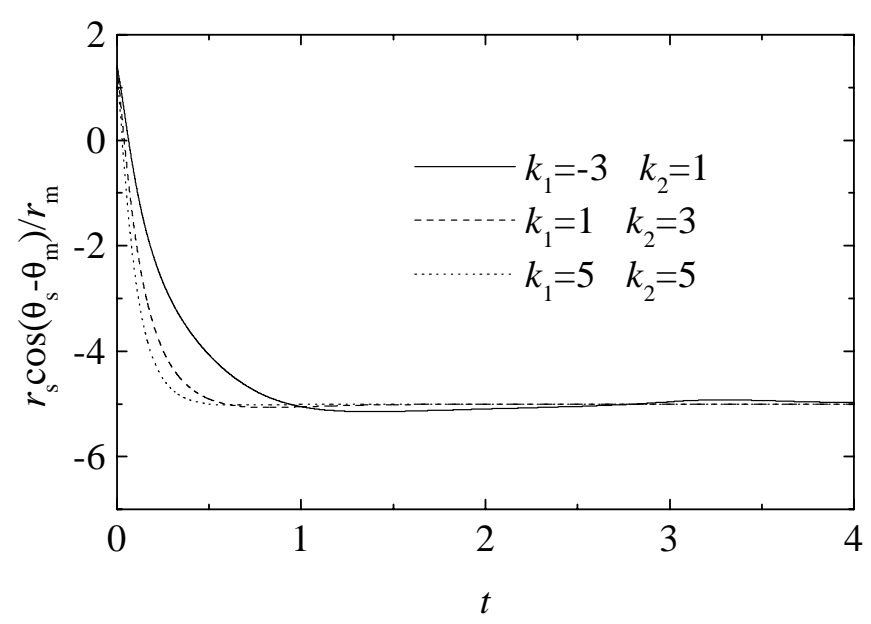

(d)

Fig. 2. Controlled PS for the Rössler system $(a=0.398, b=2$ and $c=4)$. (a) Synchronized trajectories (in $x-y$ plane) with an assigned scaling factor $\alpha^{*}=5$, (b) the variation of the ratio with distinct convergence rates, (c) synchronized trajectories (in $x-y$ plane) with $\alpha^{*}=-5$, (d) the variation of the ratio. Initial condition is $\left\{x_{m}, y_{m}, z, x_{s}, y_{s}\right\}=\{0.1,0.2,0.1,0.3,0.2\}$.

shown by the curves with different values of $k_{1}$ and $k_{2}$ in Figs. 2(b) and 2(d). It is easy to find that the convergence time of the control is decreased with the augmentation of $k_{1}$ and $k_{2}$, in accordance with the analysis mentioned above.

The second example on the implementation of the control method is provided in Chua's circuit [Chua, 1993] defined in a cubic nonlinear form

$$
\begin{aligned}
& \dot{x}=\rho \varepsilon\left(y-a x^{3}-c x\right), \\
& \dot{y}=\rho(x-y+z), \\
& \dot{z}=\rho(-\beta y-\gamma z) .
\end{aligned}
$$

For this system, Eq. (8) can be written as

$$
\begin{aligned}
\dot{V}= & \left(\varepsilon e_{2}-\varepsilon c e_{1}+\varepsilon a x_{s}^{3}-\alpha^{*} \varepsilon a x_{m}^{3}-v_{1}\right) e_{1} \\
& +\left(\left(\alpha^{*}-1\right) z+e_{1}-e_{2}-v_{2}\right) e_{2} .
\end{aligned}
$$

Similarly, we set

$$
\left\{\begin{aligned}
v_{1}= & \varepsilon e_{2}+\varepsilon a x_{s}^{3}-\alpha^{*} \varepsilon a x_{m}^{3} \\
& +k_{1}\left(\alpha^{*} x_{m}-x_{s}\right) \\
v_{2}= & \left(\alpha^{*}-1\right) z+\left(\alpha^{*} x_{m}-x_{s}\right), k_{1}>-\varepsilon c, k_{2}>-1 \\
& +k_{2}\left(\alpha^{*} y_{m}-y_{s}\right)
\end{aligned}\right.
$$

such that $\dot{V}=-\left(k_{1}+\varepsilon c\right) e_{1}^{2}-\left(k_{2}+1\right) e_{2}^{2}<0$, indicating that $\lim _{t \rightarrow \infty} \mathbf{e}(t)=0$ and PS occurs.

The controlled PS of Chua's circuit is shown in Fig. 3 with two assigned scaling factors $\alpha^{*}=5$ in Figs. 3(a) and 3(b), $\alpha^{*}=-5$ in Figs. 3(c) and 3(d). In Fig. 3, the parameters are set as $\rho=1, \varepsilon=10$, $a=1, \beta=16, c=-0.143, \gamma=0$ with the initial conditions $\left\{x_{m}, y_{m}, z, x_{s}, y_{s}\right\}=\{-0.074,0.023$, $0.062,-0.05,0.03\}$. We can see that PS happens after a short transient under the control (14) and 


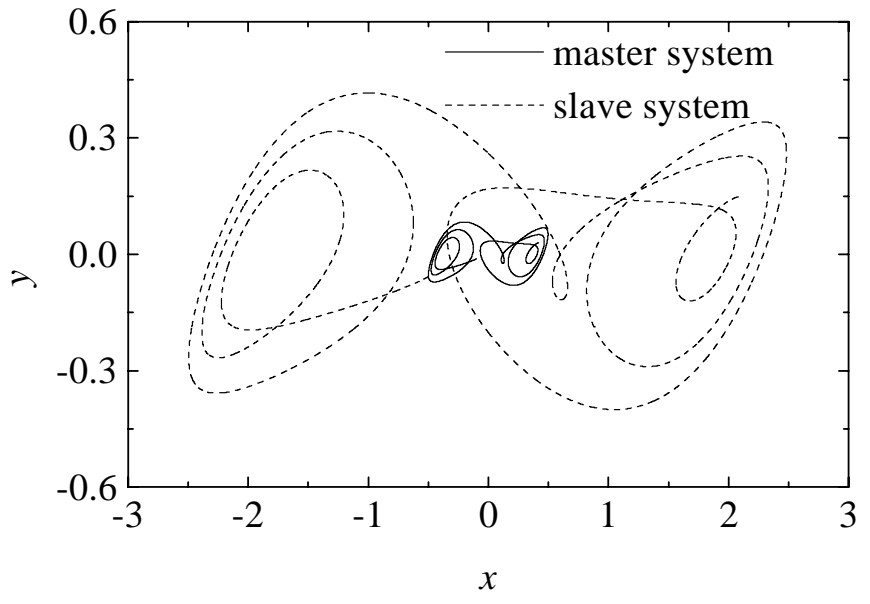

(a)

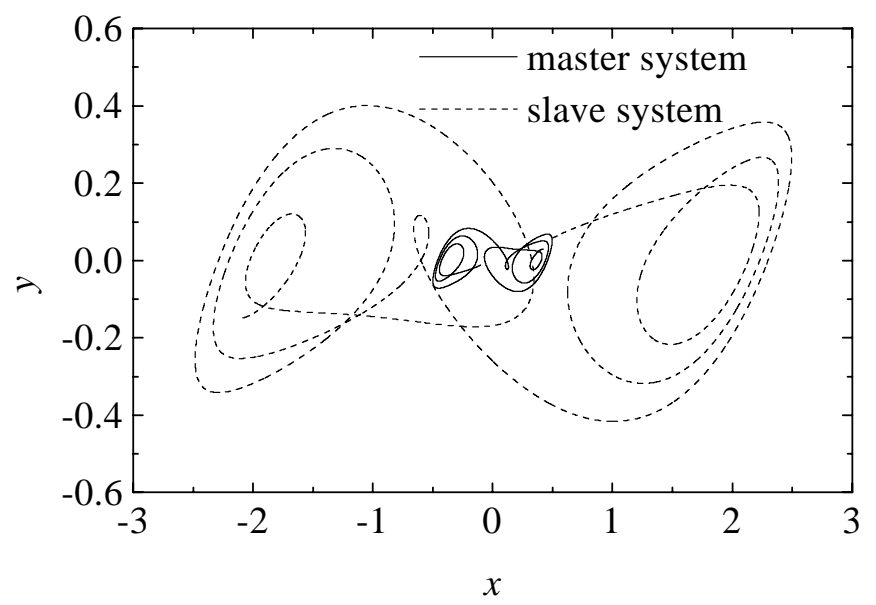

(c)

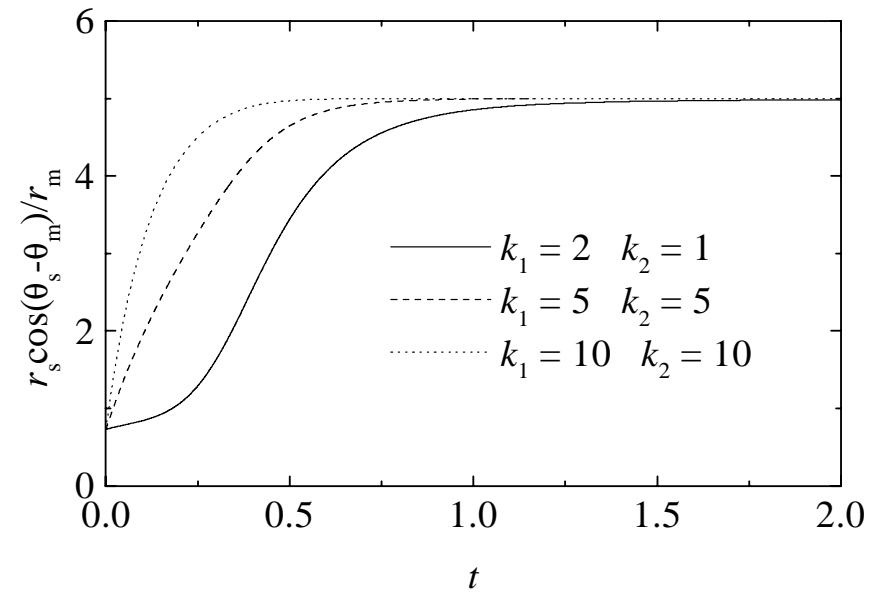

(b)

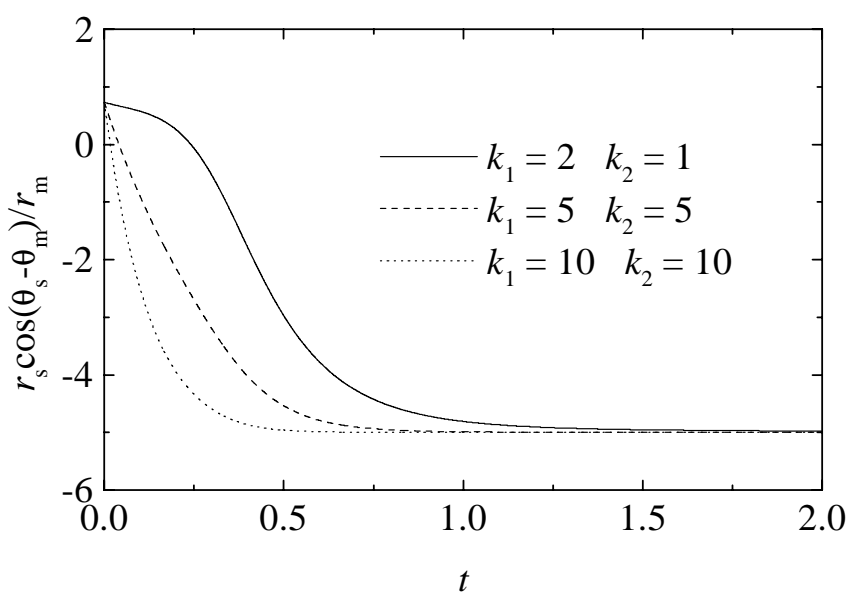

(d)

Fig. 3. Controlled PS for Chua's circuit $(a=1, \rho=1, \varepsilon=10, \beta=16, c=-0.143$ and $\gamma=0)$. (a) Synchronized trajectories with an assigned scaling factor $\alpha^{*}=5$, (b) the evolutions of the ratio $r_{s} \cos \left(\theta_{s}-\theta_{m}\right) / r_{m}$, (c) synchronized trajectories with an assigned value $\alpha^{*}=-5$ and (d) the evolutions of the ratio $r_{s} \cos \left(\theta_{s}-\theta_{m}\right) / r_{m}$. Initial condition is set as $\left\{x_{m}, y_{m}, z, x_{s}\right.$, $\left.y_{s}\right\}=\{-0.076,0.009,0.122,-0.05,0.03\}$.

the scaling factors are directed onto the desired values $\left[\alpha^{*}=5\right.$ in Fig. 3(b) and $\alpha^{*}=-5$ in Fig. 3(d)]. The effects of $k_{1}$ and $k_{2}$ on the control are displayed in Figs. 3(b) and 3(d).

\section{Discussions}

The previous two examples provided are the cases of three-dimensional systems. Theoretically, the control method could be applied to high dimensional systems. It seems always possible to find a control vector to enable the derivative of Lyapunov function $V$ to be negative. As an example for high dimensions, we apply the control to the hyperchaotic
Rössler system [Rössler, 1976]

$$
\begin{aligned}
\dot{x} & =-y-z, \\
\dot{y} & =x+a y+w, \\
\dot{z} & =b+x z, \\
\dot{w} & =c z+d w,
\end{aligned}
$$

where the parameters are set as $a=0.25, b=3$, $c=-0.5, d=0.05$. For the coupled system (15), $\mathbf{u}=(x, y, z), \mathbf{e}=\left(e_{1}, e_{2}, e_{3}\right), \mathbf{v}=\left(v_{1}, v_{2}, v_{3}\right)$ with $w$ as the coupling variable. The occurrence of PS in the system (15) could be realized by setting the control vector as 


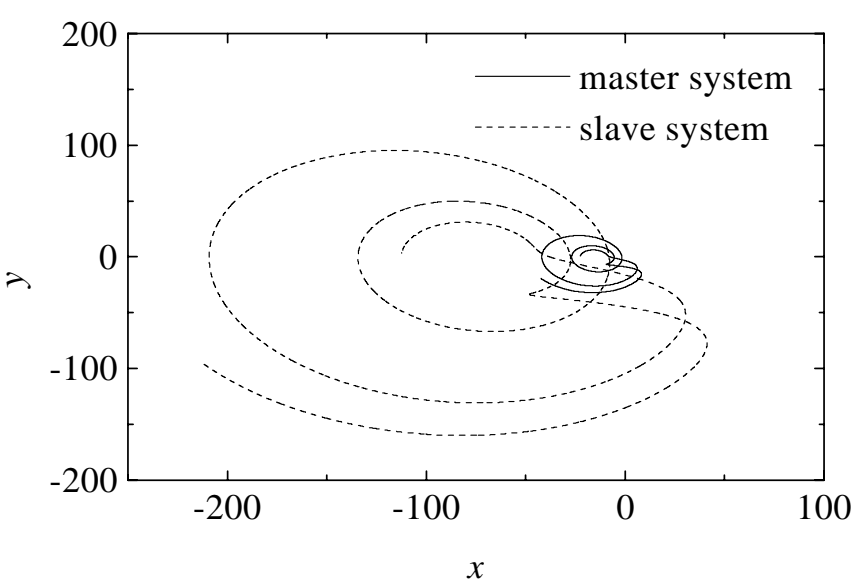

(a)

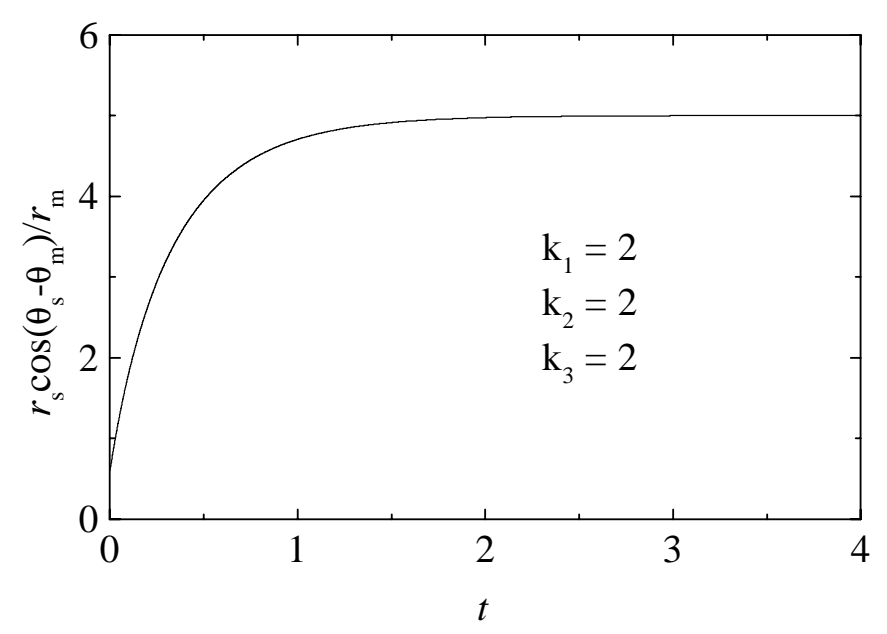

(b)

Fig. 4. Controlled PS for the hyperchaotic Rössler system $(a=0.25, b=3, c=-0.5, d=0.05)$ with the desired scaling factors $\alpha^{*}=5$. Initial condition is set as $\left\{x_{m}, y_{m}, z_{m}, w, x_{s}\right.$, $\left.y_{s}, z_{s}\right\}=\{-10,20,20,30,10,20,10\}$; (a) two synchronized trajectories (in $x-y$ plane) move with the same phase angles, (b) the evolutions of the ratio $r_{s} \cos \left(\theta_{s}-\theta_{m}\right) / r_{m}$, which tends to $\alpha^{*}=5$.

$$
\left\{\begin{aligned}
v_{1}= & -e_{2}-e_{3}+k-1 e_{1} \\
v_{2}= & e_{1}+\left(\alpha^{*}-1\right) w+k_{2} e_{2}, k_{1}>0, k_{2}>-a, k_{3}>0 \\
v_{3}= & \alpha^{*} x_{m} z_{m}-x_{s} z_{m} \\
& +\left(\alpha^{*}-1\right) b+k_{3} e_{3}
\end{aligned}\right.
$$

A controlled PS is displayed in Fig. 4 with a designed scaling factor $\alpha^{*}=5$.

In conclusion, we have introduced a control method for developing PS in nonpartially linear systems. The method allows us to manipulate the response dynamics in a duplicated pattern with any scalar multiple of the driving dynamics. To illustrate the control algorithm, we applied it to the Rössler system and the Chua's circuit. The effectiveness of the method for high dimensional chaotic systems is demonstrated in the hyperchaotic Rössler system. The numerical results indicate that PS can be extended to general classes of coupled nonlinear chaotic systems with a desired scaling factor by adding a control vector to the response system. The control vector is determined based on the Lyapunov stability theory, guaranteeing the global stability of the control.

\section{References}

Chua, L. O. [1993] "Global unfolding of Chua's circuit," IEICE Treans. Fund. Electron. Comm. Comput. Sci. E76-A(5), 704-734.

Cuomo, K. M. \& Oppenheim, A. V. [1993] "Circuit implementation of synchronized chaos with applications to communications," Phys. Rev. Lett. 71, 65-68.

Hayes, S., Grebogi, C. \& Ott, E. [1993] "Communicating with chaos," Phys. Rev. Lett. 70, 3031-3034.

Hayes, S., Grebogi, C., Ott, E. \& Mark, A. [1994] "Experimental control of chaos for communication," Phys. Rev. Lett. 73, 1781-1784.

Kocarev, L., Halle, K. S., Eckert, K., Chua, L. O. \& Parlitz, U. [1992] "Experimental demonstration of secure communications via chaotic synchronization," Int. J. Bifurcation and Chaos 2, 709-713.

Kocarev, L. \& Parlitz, U. [1996] "Generalized synchronization, predictability, and equivalence of unidirectionally coupled dynamical systems," Phys. Rev. Lett. 76, 1816-1819.

Li, Z. \& Xu, D. [2001] "Stability criterion for projective synchronization in three-dimensional chaotic systems," Phys. Lett. A282, 175-179.

Lorenz, E. N. [1963] "Deterministic non-periodic flow," J. Atmos. Sci. 20, 130-141.

Mainieri, R. \& Rehacek, J. [1999] "Projective synchronization in three-dimensional chaotic systems," Phys. Rev. Lett. 82, 3042-3045.

Parlitz, U. [1996] "Estimating model parameters from time series by autosynchronization," Phys. Rev. Lett. 76, 1232-1235.

Pecora, L. M. \& Carroll, T. L. [1990] "Synchronization in chaotic systems," Phys. Rev. Lett. 64, 821-824.

Roman, F. S. S, Boccaletti, S., Maza, D. \& Mancini, H. [1998] "Weak synchronization of chaotic coupled map lattices," Phys. Rev. Lett. 81, 3639-3642.

Rosenblum, M., Pikovsky, A. \& Kurtz, J. [1996] "Phase synchronization of chaotic oscillators," Phys. Rev. Lett. 76, 1804-1807.

Rössler, O. E. [1976] "An equation for hyperchaos," Phys. Lett. A71(2\&3), 155-157. 
Rulkov, N. F., Sushchik, M. M., Tsimring, L. S. \& Abarbanel, H. D. I. [1995] "Generalized synchronization of chaos in directionally coupled chaotic systems," Phys. Rev. E51, 980-994.

$\mathrm{Xu}, \mathrm{D}$. [2001] "Control of projective synchronization in chaotic systems," Phys. Rev. E63, 27201-27204.
Xu, D., Li, Z. \& Bishop, S. R. [2001] "Manipulating the scaling factor of projective synchronization in three-dimensional chaotic systems," Chaos 11, 439-442. 